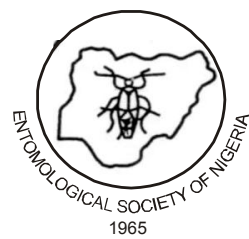

\title{
Molecular Characterization and Insecticide Susceptibility Status of Anopheles gambiae Complex (Giles, 1902) in Osun State, Southwestern Nigeria
}

\author{
Adeleke, M. A., ${ }^{1}$ Adeyemi, J. A., ${ }^{2}$ Fasasi, K. A., ${ }^{1}$ Oforka, L. C., ${ }^{3}$ Adeogun, A. O., ${ }^{4}$ \\ and Olatunde, G. O. ${ }^{1}$ \\ ${ }^{1}$ Department of Biological Sciences, Osun State University, Osogbo, Nigeria \\ ${ }^{2}$ Department of Biology, Federal University of Technology, Akure. \\ ${ }^{3}$ Department of Zoology, University of Lagos, Nigeria \\ ${ }^{4}$ Nigerian Institute of Medical Research, Yaba, Lagos, Nigeria
}

\begin{abstract}
A major challenge facing malaria control today is the high spate of insecticide resistance in mosquito populations. The present study was designed to investigate the susceptibility of An. gambiaes.l. to Dichlorodiphenyltrichloroethane (DDT) and Deltamethrin, and determine the sibling species at selected locations in Osun State, Nigeria. Larvae of An. gambiae complex were collected from Ibokun, Ife, Ede, Osogbo, Okuku and Iwo representing the three geopolitical zones in Osun State. Two to three day old unfed female mosquitoes were subjected to bioassays using papers impregnated with $0.05 \%$ Deltametrin (pyrethroid) and 4\% DDT (organochlorine) in accordance with WHO protocols. Knock down was recorded every 10 minutes for one hour and mortality measured after 24 hours. The mosquitoes were then identified using morphological keys and Polymerase Chain Reaction (PCR) assay. The results of the knockdown effects of the insecticides ranged from $40-70 \%$ in mosquitoes exposed for Deltamethrin and $15-45 \%$ for DDT across all locations. The post-exposure mortality caused by the two insecticides varied significantly; and mortality recorded in mosquitoes exposed to Deltamethrin was significantly higher than DDT $(\mathrm{p}<0.05)$ in all the study locations. All the mosquitoes exposed were highly resistant to DDT across all locations ( $0 \%-66.7 \%$ mortality). However, mosquitoes from Ede were susceptible to deltamethrin (100\% mortality), and those from Ife and Osogbo were suspected to be resistant to deltamethrin with $95 \%$ and $90 \%$ mortalities respectively while the Anopheles mosquitoes from Okuku and Ibokun were resistant to the insecticide (with $79 \%$ and $75.6 \%$ mortalities respectively). Molecular identification showed that An. gambiae s.s was the only species found across all localities except for Osogbo and Ibokun where An. Arabiensis and An. gambiae s.s were detected. The high resistance of the mosquito populations to DDT and the suspected resistance to Deltametrin indicate that DDT may not be suitable for vector control activities in Osun State while close monitoring of deltamethrin resistance is required to inform vector control interventions in the state.
\end{abstract}

Keywords: Anopheles, Deltamenthrin, DDT, Osun State, Resistance.

\section{INTRODUCTION}

Anopheles gambiae complex has been noted for its role in the transmission of deadly and

*Correspondence email: kemiteslim@yahoo.com life- threatening diseases such as malaria, bancroftian filariasis and few viruses (Adeleke et al., 2013; Aigbodion and Osariyekemken, 2013). This group of Anopheles mosquitoes had 
received serious attentions after the discovery of inconsistent biting and feeding behaviours of the members of the complex (Adeleke et al., 2008). It was later realized that the complex is made up of six genetically isolated but morphologically indistinguishable siblings with varying ecological requirements and vectorial capacities. The siblings which include $A n$. gambiae sensu stricto, An. arabiensis, An. quadrunnalatus, An. melas, An. merus and An. bwambwe could only be identified through molecular techniques (Awolola et al., 2005; Oyewole and Awolola, 2006; Noutcha and Anumudu, 2009).

A major challenge currently facing malaria control is the high spate of insecticide resistance in mosquito populations. Studies in different zones of Africa have reported the widespread DDT resistance in mosquitoes from various countries (Akogbeto and Yakoubou, 1999; Kristans et al., 2003). The existence of DDT and pyrethroid resistantAnopheles vectors has also been recorded in Nigeria (Kristian et al., 2003; Awolola et al., 2002; Awolola et al., 2005). The increased spread of insecticide resistant mosquitoes was associated with uncontrolled use of insecticides which has inundated many communities in Nigeria (Oduola and Awe, 2006). This attitude most often culminates in selected resistant mosquito populations which frustrates vector control interventions like the use of long lasting insecticide-treated bed nets (Awolola et al., 2005).

Sequel to the observed differences in vectorial capacities of members of the $A n$. gambiae complex and the increased rate of insecticides, efforts had been directed toward identifying and documenting the temporal and spatial variation in siblings and insecticide susceptibility status of the members of $A n$. gambiae complex in different localities in Nigeria (Awolola et al., 2005; Adeleke et al.,
2008). The dividends of such efforts will provide information on species composition and the distribution of insecticide resistant populations of $A n$. gambiae complex in different localities. The results will thus guide policy makers in malaria control strategies on the types of insecticides to be selected for mosquito control in different localities as Nigeria commences full integrated vector control measures.

Previous researchers had made efforts to document the sibling composition and the distribution of insecticide susceptibility status of $A$. gambiae complex in some states in Nigeria (Awolola et al., 2002; 2005; Kristian et al., 2003; Oduola et al., 2010). However, the distribution of sibling species and insecticide susceptibility status of An. gambiae complex is still unknown in many States including Osun State. The present study was therefore designed to investigate the susceptibility status of $A n$. gambiae complex to DDT and Deltamethrin at selected locations in Osun State, Nigeria and determine the sibling composition of $A$. gambiae complex in the state using molecular techniques.

\section{MATERIALS AND METHODS}

\section{Study Area}

Osun State lies between latitude $7^{\circ} 30^{\prime}$ North and $4^{0} 30^{\prime}$ East in the derived Savanna Zone of southwestern Nigeria. The state shares boundary with Oyo, Ogun, Ekiti and Kwara States. Majority of residents are farmers and engaged in the cultivation of various cash and root crops. The detailed description and map of the study area have been earlier been provided by Ojurongbe et al. (2015). Most of the roads are without drainages and where drainages exist, some of them have been blocked, therefore providing stagnant water suitable for the breeding of mosquitoes. 


\section{Collection of Mosquito Larvae and Maintenance of Adult Mosquitoes}

The larvae of $A n$. gambiae complex were collected between May and October, 2016 from six selected locations in the three geopolitical zones namely Osun East (Ibokun and Ife), Osun Central (Osogbo and Okuku) and Osun West (Iwo and Ede). Ife, Ede Osogbo and Iwo are urban areas while Okuku and Ibokun are rural communities. At each site, the water was scooped with the aid of plastic scooper and transferred to transparent plastic bowls. The larvae were transported to the Zoology Laboratory in the Department of Biological Sciences, Osun State University, Osogbo, Nigeria. The larvae were allowed to emerge into adults for insecticide bioassay. The mosquitoes were maintained in the laboratory at $27 \pm 2{ }^{\circ} \mathrm{C}$ and $72 \pm 1 \%$ relative humidity.

\section{Insecticide Susceptibility Bioassays}

The insecticide susceptibility test was carried out in accordance with WHO procedures (WHO, 2013). Two to three day old unfed female mosquitoes were exposed to papers impregnated with $0.05 \%$ Deltametrin (pyrethroid) and 4\% DDT (organochlorine) while the control was exposed to mineral oil. The bioassays were carried out on 25 mosquitoes in 3-4 replicates and 25 mosquitoes in two replicates in control. The mosquitoes were exposed to the impregnated papers for 60 minutes and the number of mosquitoes knock down (mosquitoes that fell on the base of the tube and unable to fly around within the 60 minutes of exposure) was recorded every 10 minutes during the 1 hour exposure time. The mosquitoes were thereafter transferred to holding tubes and fed with sugar solution. The final mortality was recorded after 24 hours.

\section{Morphological Identification of the Mosquitoes}

The adult mosquitoes were morphologically identified using the keys described by Gillies and Coetzee (1987) with the aid of dissecting microscope. The identified mosquitoes were preserved in $1.5 \mathrm{ml}$ eppendorf tubes containing silica gel for molecular analysis.

\section{Molecular Characterization of Siblings Species}

Species identification of Anopheles gambiae complex was carried out using Polymerase Chain Reaction (PCR). The DNA was extracted by macerating the leg of the mosquitoes on $50 \mu 1$ of STE buffer $(0.1 \mathrm{M} \mathrm{NaCl}, 10 \mathrm{Mm}$ Tris- $\mathrm{HCl}$ pH8, EDTA pH8). The eppendorf tubes containing the macerated mosquitoes were incubated at $95^{\circ} \mathrm{C}$ for $12 \mathrm{~min}$ in a heating block. The mix was centrifuge (Biorad) at 13,000 rpm for 4 mins. The supernatant (DNA) was kept in $1.5 \mathrm{ml}$ eppendorf in $4^{\circ} \mathrm{C}$ till use. The $2 \mu \mathrm{l}$ of the supernatant was added to $18 \mu 1$ of reaction mixture of dNTPs, Taq DNA polymerase and the specific primers as previously described by Scott et al. (1993).

\section{Data Analyses}

The data were subjected to t-test to determine the significant difference in knockdown effect recorded against each insecticide using SPSS version 17.0. Data were square root-transformed $\sqrt{x+0.5}$ before analyses. The criteria defined by WHO (2013) were used in classifying the mortality rates into resistance and susceptibility. The mortality rate greater than $98 \%$ indicates susceptibility while mortality between $97-90 \%$ indicates possibility of resistance.

\section{RESULTS}

\section{Knockdown effects and mortality of An. gambiae complex exposed to DDT and Deltamethrin}

Mosquito larvae were recovered from all the breeding sites visited. The larvae were reared to adults and assayed for insecticide 
susceptibility test. Table 1 shows the locations and the number of emergent adult mosquitoes for bioassay. However, all the mosquitoes for bioassay and control at Iwo died before 24 hours post-exposure. All attempts to rear mosquitoes from the site proved abortive. The dead mosquitoes were preserved for molecular analysis; but the results for insecticide susceptibility were subsequently discarded.

The results of the knockdown effects of the insecticides within 60 min of exposure ranged from $40-70 \%$ in mosquitoes exposed to Deltamethrin and $15-45 \%$ for the mosquitoes exposed to DDT across the locations. The knockdown effect was $50 \%$ and above in mosquitoes exposed to Deltamethrin in all the locations except at Ife where a knockdown of $40 \%$ was recorded. None of the mosquitoes exposed to DDT in all the locations achieved knockdown of $50 \%$ within 60 minutes of exposure (Figure 1).

Table 1: The study locations and the emergent adults of An. gambiae s.l for bioassay

\begin{tabular}{lllll}
\hline S/N & Locations & Longitude (E) & Latitude (N) & $\begin{array}{l}\text { No of Adult } A n . \\
\text { gambiae s.l for } \\
\text { bioassay }\end{array}$ \\
\hline 1 & Ede & $7^{\circ} 74^{\prime}$ & $04^{\circ} 44^{1}$ & 196 \\
2 & Ibokun & $7^{\circ} 76^{\prime}$ & $04^{\circ} 57^{1}$ & 194 \\
3 & Ife & $07^{\circ} 52^{\prime}$ & $04^{\circ} 52^{1}$ & 237 \\
4 & Okuku & $08^{\circ} 01^{\prime}$ & $04^{\circ} 67^{1}$ & 251 \\
5 & Osogbo & $07^{\circ} 78^{\prime}$ & $04^{\circ} 57^{1}$ & 295 \\
6 & Iwo & $07^{\circ} 63^{\prime}$ & $04^{\circ} 17^{1}$ & 69 \\
\hline
\end{tabular}

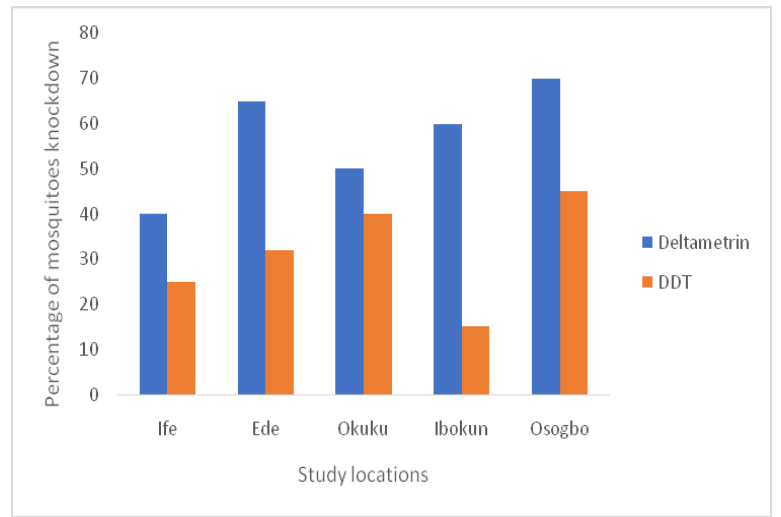

Fig. 1: Percentage of mosquito knockdown after one-hour exposure to insecticides

The post-exposure mortality caused by the two insecticides varied significantly; and the mortality recorded in mosquitoes exposed to Deltamethrin was significantly higher than DDT $(\mathrm{P}<0.05)$ in all the study locations. The mortality recorded in mosquitoes exposed to DDT was less than $80 \%$ in all the locations. Zero mortality was recorded in Ibokun while the mortality in other sites ranged from $50-66.7 \%$. All the mosquitoes exposed to Deltamethrin in Ede were susceptible to the insecticide (100\% mortality). The mortalities in mosquitoes exposed to Deltamethrin in Osogbo and Ife were $90 \%$ and $95 \%$ respectively; while the percentage mortality was less than $80 \%$ in Ibokun and Okuku (Table 2).

\section{Molecular characterization of sibling composition of $A n$. gambiae complex at the study sites}

The data obtained on species composition of the An. gambiae complex are presented in Figure 2. Two sibling species of An. gambiae complex were encountered; An gambiae s.s and 
Table 2: Post exposure mortality of the mosquitoes to DDT and Deltamethrin after 24 hours

\begin{tabular}{lll}
\hline Locations & $\begin{array}{l}\text { Mortality (\%) } \\
\text { DDT }\end{array}$ & DEL \\
\hline Okuku & 66.7 & 79.0 \\
Osogbo & 50.0 & 90.0 \\
Ife & 50. & 95.0 \\
Ibokun & 0.0 & 75.6 \\
Ede & 65.3 & 100 \\
\hline
\end{tabular}

$\mathrm{P}<0.05$; DEL: Deltamethrin; DDT:

dichlorodiphenyltrichloroethane

An. arabiensis. All An. gambiae complex assayed in Ife, Iwo, Ede and Okuku were $A$. gambiae s.s. However, An. arabiensis constituted $9.09 \%$ and $16.8 \%$ of mosquitoes assayed in Osogbo and Ibokun respectively; others belong to An. gambiaes.S

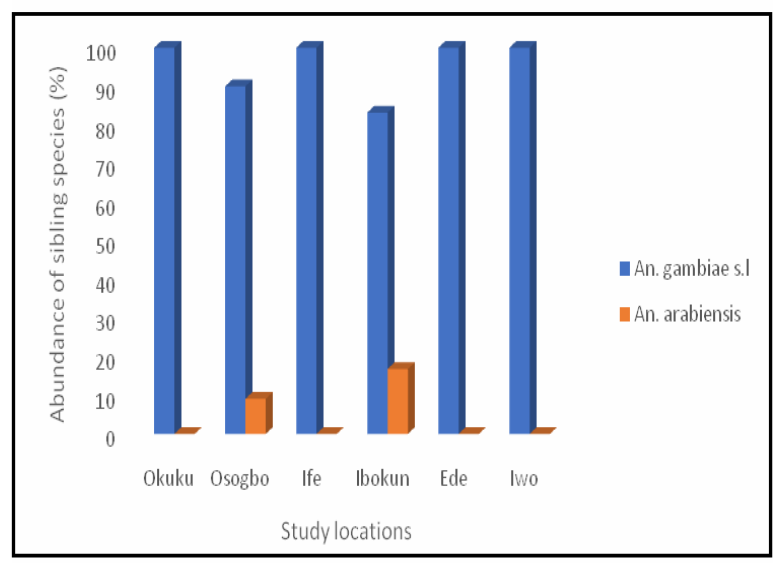

Fig. 2: Composition of siblings species of Anopheles gambiae complex at the study area

\section{DISCUSSION}

The results of the present study showed preponderance of An. gambiaes.s in all the study locations except Osogbo and Ibokun where sympatric existence of An. gambiaes.s and An. arabiensis exist. Contrary to earlier speculation that the distribution of $A n$. gambiaes.s and An. arabiensis is dependent of rural-urban drift in Southwestern Nigeria (Kristian et al., 2003), with the former adapting to rural areas while the later predominating in urban areas; the pattern observed in the present study is not suggestive of this speculation as occurrence of An. gambiae s.s was exclusively found in urban (Ife, Ede and Iwo) and rural (Okuku) areas. The co-existence of both siblings was equally recorded in urban (Osogbo) and rural area (Ibokun). Our observations are in consonance with earlier reports in Oyo State where preponderance of An. gambiae s.s was recorded in both rural and urban areas (Oduola et al., 2010).

Earlier studies in three rural communities in Obokun Local Government Area (LGA), Osun State had reported the predominance of An. gambiae s.s (95\%) over An. arabiensis (5\%) (Oduola et al., 2013a). Coincidentally, Ibokun (one of our study sites) is the Headquarter of Obokun Local Government where dominance of An. gambiaes.s (83.2\%) was also observed over An. arabiensis (16.8\%). Unlike previous study which was restricted to three rural communities in a LGA, the present study spread across six LGAs representing urban and rural niches in the three geo-political senatorial areas of Osun State. To the best of our knowledge based on available literature, this is the first report on spatial distribution of sibling species of An. gambiae complex in Osun State, Nigeria.

The factors responsible for the distribution of the sibling species in the study areas are unclear; however, few speculations could be adduced to explain the pattern observed. Several authors had identified ecological factors as driving force for the distribution of sibling species of An. gambiae complex in West Africa (Onyabe and Conn, 2001; Awolola et al., 2005; Oyewole and Awolola, 2006, Noutcha and Anumudu, 2009). An. arabiensis had been reported to be well adapted to savannah arid zones while An. gambiae s.s prefers humid and forested regions. Since Osun 
State Nigeria is the rain forest/ derived savannah belt, this could have accounted for the dominance of An. gambiae s.s in the study locations. Moreover, the sympatric existence of An. arabiensis and An. gambiae in Ibokun and Osogbo may suggest the spatial spread of the siblings; as Ibokun is an adjoining rural community to Osogbo. These speculations, however, should be the thrust of hypotheses for further studies.

The results of insecticide susceptibility bioassays suggest wide spread of resistance of mosquitoes to Deltamethrin and DDT in Osun State. Apart from Ede where the mosquitoes were completely susceptible to Deltamethrin, the mosquitoes were resistant to the insecticide in other locations. The values obtained from the number of mosquito knockdown within 60 minutes of exposure and susceptibility bioassays of mosquitoes to Deltamethrin and DDT in the present study were by far lower than the values recorded by Awolola et al (2002) in some parts of Nigeria and Kristan et al (2003) in Ogun State. Similar observations had also been recently reported by Oduola et al (2010) and Oduola et al (2013b) in which the resistance of mosquitoes to DDT had been observed in different zones in Nigeria.

Statistical analysis revealed that the mortality recorded against Deltamethrin was significantly higher than DDT across the five study locations. Even though, the wide spread and selection of resistance genes for pyrethroid based insecticides by the mosquitoes had been premised on the indiscriminate use of agricultural insecticides (Akogbeto and Yakoubou, 1999), the factors accounting for high spate selection of resistance to DDT are still largely unclear in Nigeria despite the ban on its use in Nigeria. It may, therefore, be tempting to infer that the illegal use of DDT may be ongoing in both agricultural and nonagricultural areas in the State. Observation on resistance of mosquitoes to DDT had also been reported in non-agricultural areas in Coted'Ivore (Tia et al., 2006). On the other hand, the role of indiscriminate use of locally made insecticides in insecticidal resistance selection mechanisms could not be underestimated. The Nigerian markets are in recent time inundated with indiscriminate sale of locally made insecticides whose chemical constituents are largely unknown but enjoy wide patronage by the people. These factors, either singly or in synergy may be the reasons to explain the resistance of An. gambiae s.1 to Deltamethrin and DDT in the study locations since agriculture is the main occupation of the majority of the residents of Osun State and the tendency of indiscriminate use of both agricultural and nonagricultural insecticides could not be downplayed in the area.

The results obtained in the present study have profound implications in vector control strategies/policies in Osun State. The impregnation of the insecticide treated bednets relies on pyrethroid based insecticides such as Deltamethrin (Kristian et al., 2003). Moreover, World Health Organization had also approved the use of DDT for Indoor Residual Spraying (IRS) activities (WHO, 2006) and Osun State is currently proposing scale up campaign on IRS as one of the viable options in the control and management of malaria in the State. Therefore, the use of the two insecticides (Deltamethrin and DDT) for vector control and management in Osun State may not yield any appreciable results as the local populations of An. gambiae complex are resistant to the two insecticides virtually in all the study locations and the cross resistance of organochlorine and pyrethroid based insecticide had been well reported in many locations in Africa (Oduola et al., 2013a).

In conclusion, the results of the present study showed that the populations of $A n$. 
gambiae complex are resistant to DDT and Deltamethrin in all the study locations, except Ede where the population was susceptible to Deltamethrin. Molecular characterization of the An. gambiae complex showed the dominance of An. gambiae s.s and sympatric existence of An. arabiensis and An. gambiae s.s in Osogbo and Ibokun. Based on the foregoing, it is recommended that the policies on the malaria vector control strategies in Osun State should be guided by scientific evidence on the susceptibility of local populations of Anopheles mosquitoes to various classes of insecticides approved by WHO, and that there should be further studies on the susceptibility of local mosquito vectors to other classes of WHO approved insecticides in the State.

\section{ACKNOWLEDGEMENTS}

The authors wish to thank the field scientists that assisted in carrying out this study. We thank Dr. Sam Awolola for the logistic support. This project was fully funded under Tertiary Education Trust Fund (TETFUND-UNIOSUN/ 2013-2014/002) for Institutional research by the government of Federal Republic of Nigeria.

\section{REFERENCES}

Adeleke, M.A., Mafiana, C.F., Idowu, A.B., Amusan, A.A.S. and Adekunle, M.F. (2008). Morphometric Studies on Anopheles gambiae complex (Diptera: Culicidae) in Abeokuta, Southwest Nigeria, Acta Entomological Sinica, 51(12): 1289-1292.

Adeleke, M.A., Adebimpe, W.O., Hassan, A.O., Oladejo, S.O., Olaoye., I.K., Olatunde, G.O. and Adewole, T.A. (2013). Larval ecology of mosquito fauna in Osogbo, Southwestern Nigeria, Asian-Pacific Journal of Tropical Biomedicine 3(9): 673-677..

Aigbodion, F.I. and Osariyekemken, O.U. (2013). Temporal distribution and habitat diversification by some mosquitoes (Diptera: Culicidae) species in Benin-City Nigeria. Journal of Entomology, 10: 13-23.
Akogbeto, M. and Yakoubou, S. (1999). Resistance of malaria vectors to pyrethroids used for impregnated bednets in Benin, West Africa. Bulletin de la Societe de Pathologie Exotique, 92, 123-130.

Awolola, T.S., Oyewole, I.O., Amajoh, C.N., Idowu, E.T., Ajayi, M.B., Oduola, A.O., Manafa, O.U., Ibrahim, K., Koekemoer, L.L. and Coetzee, M. (2005). Distribution of the molecular forms of Anopheles gambiae and pyrethroid knock down resistance gene in Nigeria, Acta Tropica, 95(3): 204-209.

Awolola, T.S., Brooke, B.D., Hunt, R.H. and Coetzee, M. (2002). Resistance of the malaria vector Anopheles gambiae s.s to pyrethroid insecticides in Southwestern Nigeria. Annals of Tropical Medicine and Parasitology, 96, 849-852.

Gillies M. T. and Coetzee M. A. (1987). Supplement to the Anophelinae of Africa South of the Sahara. In Publication of South African Institute of Medical Research, 55: 1143.

Kristan, M., Fleischmann, H., Della-Torre, A., Stich, A. and Curtis, C.F (2003). Pyrethroid resistance/susceptibility and differential urban/ rural distribution of Anopheles arabiensis and Anopheles gambiae s.s malaria vectors in Nigeria and Ghana. Medical and Veterinary Entomology, 17, 326332.

Noutcha, M.A.E. and Anumdu, C.I. (2009). Entomological Indices of Anopheles gambiae Sensu lacto at a rural community in Southwestern Nigeria. Journal of Vector Borne Diseases, 46: 43-51.

Oduola, O.A. and Awe, O.O. (2006). Behavioural biting preference of Culex quinquefasciatus in human host in Lagos Metropolis Nigeria, Journal of Vector Borne Diseases, 43: 16-20.

Oduola, A.O., Obansa, J.B. Ashiegbu, C.O., Adeogun, A., Otubanjo, O.A. and Awolola, T.S. (2010). High level of DDT resistance in the malaria mosquito: Anopheles gambiae s.l. from rural, semi urban and urban communities in Nigeria. Journal of Rural Tropical Public Health, 9: 114-120. 
Oduola, A.O., Idowu, E.T., Oyebola, M.K., Adeogun, A.O., Olojede., J.B., Otubanjo, O.A. and Awolola, T.S. (2013a). Evidence of carbamate resistance in urban populations of An. gambiae ss mosquitoes resistant to DDT and Deltamethrin insecticides in Lagos, Southwestern Nigeria., Parasites and Vectors, 5: 116.

Oduola, A.O., Obembe, A., Adeogun, A. and Awolola, T.S (2013b). Entomological and transmission risk indices of malaria vectors in selected communities in Osun State, Nigeria; Animal Research International, 10(3): 1805-1808.

Ojurongbe, O., Akindele, A. A., Adeleke M.A, Oyedeji M.O., Adedokun S.A., Ojo J.F., Akinleye, C.A., Bolaji, O.S., Adefisoye, O.A. and Adeyeba, O.A. (2015). Coendemicity of Loiasis and Onchocerciasis in Rain Forest Communities in Southwestern Nigeria. PLoS Neglected Tropical Diseases, 9(3):1-8.

Onyabe, D.Y. and Conn, J.E. (2001). The distribution of two major malaria vectors, Anopheles gambiae and Anopheles arabiensis in Nigeria, Memorias do Instituto Oswaldo Cruz, Rio de Janeiro, 96(8): 10811084.
Oyewole, I.O. and Awolola, T.S. (2006). Impact of urbanization on bionomics and distribution of malaria vectors in Lagos, Southwestern Nigeria. Journal of Vector Borne Diseases, 43: 172-178.

Scott, J. A., Brogdon, W.G. and Collins, F.H. (1993). Identification of single Specimen of the Anopheles gambiae complex by Polymerase Chain Reaction. American Journal of Tropical Medicine and Hygiene, 49: 520-529.

Tia, E., Akogbeto, M., Koffi, A., Toure, M. and Adja, A.M. (2006). Pyrethroid and DDT resistance of Anopheles gambiae s.s. (Diptera: Culicidae) in five agricultural ecosystems from Côte-d'Ivoire. Bulletin Sociologic Pathologic Exotica, 4: 278-82.

WHO (2006). Indoor Residual Spraying: Use of Indoor Residual Spraying for Scaling UpGlobal Malaria Control and Elimination: WHO Position Statement. Geneva:Global Malaria Programme, World Health Organization, $10 \mathrm{pp}$.

WHO (2013). Test procedures for insecticide resistance monitoring in malaria vector mosquitoes. World Health Organization; Geneva, Switzerland, 54pp.
Nigeria Journal of Entomology

Published by the Entomological Society of Nigeria

www.esnjournal.com.ng

ISSN: 0331-0094

Vol. 34, 2018; pp. 69-76

DOI:10.36108/NJE/8102/43(0180)

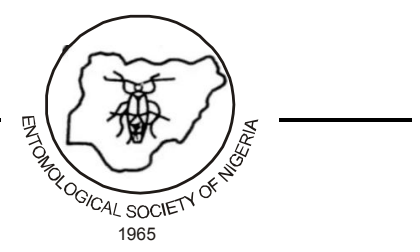

